\title{
P04-7-6 Poster session
}

\section{Study on pharmacological validity of brain ischemia-reperfusion model using zebrafish}

\author{
Mami Matsumoto ${ }^{1}$, Masahito Sawahata ${ }^{1}$, Kana Oda ${ }^{1}$, Moeri Miyamoto ${ }^{1}$, Yasuhiko Izumi ${ }^{1,2}$, \\ Toshiaki Kume ${ }^{1,3}$
}

${ }^{I}$ Department of Pharmacology, Graduate School of Pharmaceutical science, Kyoto University, Japan, ${ }^{2}$ Department of Pharmacology, Kobe Pharmaceutical University, Japan, ${ }^{3}$ Department of Applied Pharmacology, Graduate School of Medicine and Pharmaceutical Sciences, University of Toyama, Japan

We previously established new brain ischemia-reperfusion model induced by hypoxia-reoxygenation (H/R) using zebrafish larvae. In this model, H/R induced brain cell death and neuronal cell damage. Here, we investigated the pharmacological validity of this brain ischemia-reperfusion model using the reagents which showed protective effect against ischemic injury in rodent models.

We used the double-transgenic zebrafish line, Tg(flila:eGFP)/ Tg(gata1:mRFP), in which blood vessels and erythroid precursors were respectively labeled with enhanced green fluorescent protein and monomeric red fluorescent protein, or single line $\mathrm{Tg}$ (gata1:mRFP). Zebrafish larvae were examined at 4-5 days post fertilization. They were mounted by 1.2 -1.5\% low-melting agarose in E3 medium and added hypoxic E3 medium. Hypoxic condition was made by hypoxic E3 medium which was created by injecting pure nitrogen. Subsequently, reoxygenation was made by replacement from hypoxic E3 medium to normoxic E3 medium. Zebrafish larvae were imaged on confocal microscope and dead cells were stained by acridine orange. MK-801 and memantine were treated during hypoxic condition. Edaravone was treated during reoxygenation.

We validated this animal model using pharmacological experiments. Ischemia-reperfusion results in neuronal injury by the overactivation of glutamate receptors, especially NMDA receptor, and the generation of free radicals. First, we examined the effects of MK-801 and memantine, antagonists of NMDA receptor, on brain cell death induced by H/R. Treatment of MK-801 or memantine with hypoxic E3 medium prevented brain cell death induced by H/R. Next, we examined the effect of edaravone, a free radical scavenger, on brain cell death induced by $\mathrm{H} / \mathrm{R}$. Treatment of edaravone with normoxic E3 medium after removal of hypoxic E3 medium suppressed brain cell death induced by H/R. These results supported the validity of this brain ischemia-reperfusion model induced by $\mathrm{H} / \mathrm{R}$ using zebrafish larvae. 